\title{
Module derivations and cohomological splitting of adjoint bundles
}

\author{
by \\ Akira Kono (Kyoto) and Katsuhiko Kuribayashi (Okayama) \\ Dedicated to Professor Hideyuki Kachi on his 60th birthday
}

\begin{abstract}
Let $G$ be a finite loop space such that the $\bmod p$ cohomology of the classifying space $B G$ is a polynomial algebra. We consider when the adjoint bundle associated with a $G$-bundle over $M$ splits on $\bmod p$ cohomology as an algebra. In the case $p=2$, an obstruction for the adjoint bundle to admit such a splitting is found in the Hochschild homology concerning the $\bmod 2$ cohomologies of $B G$ and $M$ via a module derivation. Moreover the derivation tells us that the splitting is not compatible with the Steenrod operations in general. As a consequence, we can show that the isomorphism class of an $S U(n)$-adjoint bundle over a 4-dimensional CW complex coincides with the homotopy equivalence class of the bundle.
\end{abstract}

1. Introduction. Let $G$ be a connected finite loop space, in other words, a connected topological group with the homotopy type of a finite $\mathrm{CW}$ complex. Let $L G$ denote the loop group which is the space of free loops on $G$. In [12], the notion of the module derivation has been introduced when considering the bar type and cobar type Eilenberg-Moore spectral sequences converging to the mod $p$ cohomology algebra of the classifying space $B L G$ of $L G$. In this paper, by investigating the $\bmod p$ cohomology algebra over the Steenrod algebra of the total space of an adjoint bundle, we show that the module derivation is relevant in analyzing the first line of the bar type Eilenberg-Moore spectral sequence for some fibre square.

Let $P \rightarrow M$ be a principal $G$-bundle over a connected space $M$ and $P \times{ }_{\text {ad }} G \rightarrow M$ the adjoint bundle, namely, the bundle associated with $P \rightarrow$ $M$ via the adjoint map ad : $G \times G \rightarrow G$ defined by $\operatorname{ad}(g, h)=g h g^{-1}$. If $M=B G$ and $P \rightarrow B G$ is the universal $G$-bundle, then $P \times$ ad $G$ can be regarded as the classifying space $B L G$ of the loop group $L G$. In the category

2000 Mathematics Subject Classification: 55T20, 57T35, 55S05.

Key words and phrases: $\bmod p$ cohomological splitting, $\mathcal{A}(p)$-cohomological splitting, Hochschild homology, module derivation, the bar type Eilenberg-Moore spectral sequence. 
of differentiable manifolds, if $M$ is a finite-dimensional manifold and $G$ is a Lie group, then the bundle $P \times_{\text {ad }} G \rightarrow M$ is the object on which gauge theory is developed. We also wish to mention the theorem due to Tsukuda [18] which says that there is a one-to-one correspondence between appropriate isomorphism classes of gauge groups and those of adjoint bundles. These facts give us a motivation to investigate the topology of $P \times_{\text {ad }} G$.

In order to state our results exactly, we begin with some definitions concerning algebras. Let $A$ be a graded commutative algebra over $\mathbb{F}_{p}$ and $N$ a graded commutative algebra over $\mathbb{F}_{p}$ equipped with a left (resp. right) $A$-module structure, where $p$ is a prime or zero and $\mathbb{F}_{0}$ represents the rational number field. Then $N$ is said to be a left (resp. right) $A$-algebra if the $A$ module structure map $A \otimes N \rightarrow N$ (resp. $N \otimes A \rightarrow N$ ) is a morphism of algebras. We say that an $A$-bimodule $N$ is an $A$-bialgebra if $N$ is a right and left $A$-algebra. To simplify, we shall refer to a left $A$-algebra simply as an A-algebra.

Henceforth, it will be assumed unless otherwise stated that the $H^{*}\left(M ; \mathbb{F}_{p}\right)$-algebra structure of $H^{*}\left(E ; \mathbb{F}_{p}\right)$ is defined by composing $\pi^{*} \otimes 1$ with the multiplication on $H^{*}\left(E ; \mathbb{F}_{p}\right)$ when a map $\pi: E \rightarrow M$ is given. We say that a fibration $F \rightarrow E \rightarrow M$ is homotopy equivalent to another fibration $F \rightarrow E^{\prime} \rightarrow M$ with the same fibre and base if there exists a homotopy commutative diagram

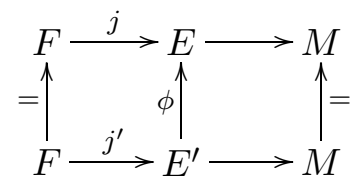

in which $\phi$ is a homotopy equivalence. Under this assumption, the induced homomorphism $\phi^{*}$ on mod $p$ cohomology is an isomorphism of $H^{*}\left(M ; \mathbb{F}_{p}\right)$ algebras and enjoys the compatibility $j^{\prime *} \circ \phi^{*}=j^{*}$. By algebraically modifying the case where $F \rightarrow E^{\prime} \rightarrow M$ is the trivial fibration $F \stackrel{\mathrm{in}_{2}}{\longrightarrow} M \times F \stackrel{\mathrm{pr}_{1}}{\longrightarrow} M$, we propose a definition of the cohomological splitting of a fibration.

Definition 1.1. A fibration $F \stackrel{j}{\rightarrow} E \rightarrow M$ admits a mod $p$ cohomological splitting if there exists an isomorphism of $H^{*}\left(M ; \mathbb{F}_{p}\right)$-algebras

$$
\phi: H^{*}\left(E ; \mathbb{F}_{p}\right) \cong H^{*}\left(M ; \mathbb{F}_{p}\right) \otimes H^{*}\left(F ; \mathbb{F}_{p}\right)
$$

such that $\operatorname{in}_{2}^{*} \circ \phi=j^{*}$. We also say that such a fibration splits on $\bmod p$ cohomology. Moreover, if the isomorphism $\phi$ is a morphism over the Steenrod algebra $\mathcal{A}(p)$, that is, $\phi$ is compatible with the action of the Steenrod operations, then we say that the fibration $F \rightarrow E \rightarrow M$ admits an $\mathcal{A}(p)$ cohomological splitting.

In what follows, the fibrations we consider are the adjoint bundles $G \rightarrow$ $P \times{ }_{\text {ad }} G \rightarrow M$ in which the $\bmod p$ cohomology of $M$ is of finite type and the 
$\bmod p$ cohomology of $B G$ is a polynomial algebra for a given prime number $p$. Firstly, we investigate the algebra structure of the mod $p$ cohomology of $P \times{ }_{\text {ad }} G$ without the action of the Steenrod operations, and obtain the following theorem.

TheOREM 1.2. If $p=0$ or $H^{*}(G ; \mathbb{Z})$ is $p$-torsion free, then every adjoint bundle $G \rightarrow P \times{ }_{\text {ad }} G \rightarrow M$ splits on $\bmod p$ cohomology.

Theorem 1.2 asserts that $\bmod p$ cohomological splittings of adjoint bundles do not depend on the equivalence classes of $G$-bundles over $M$.

In [10], Kono and Kozima proved that if $G$ is a compact simply connected Lie group, then the following three conditions are equivalent: (i) $H^{*}(G ; \mathbb{Z})$ is $p$-torsion free; (ii) the adjoint action Ad : $G \times \Omega G \rightarrow \Omega G$ defined by $\operatorname{Ad}(g, l)=g l g^{-1}$ and the second projection $\operatorname{pr}_{2}$ on $\Omega G$ induce the same homomorphism on mod $p$ cohomology; (iii) the bundle $G \rightarrow B L G \rightarrow B G$ splits on $\bmod p$ cohomology. Later Iwase [7] showed that the equivalence of the three conditions holds even if $G$ is a simply connected finite loop space and $p \neq 2$. Recently, Iwase and Kono [8] and Kuribayashi [12] have proved, by different methods, that for finite loop spaces the assertion remains true even for $p=2$. Theorem 1.2 is a generalization of the assertion that (i) implies (iii). We mention that Theorem 1.2 is proved without using the result of Kono and Kozima. Of course, the converse does not hold in general. In fact the adjoint bundle associated with the trivial $G$-bundle is also trivial for any $G$.

One may ask for a criterion for an adjoint bundle with structure group $G$ to admit a mod $p$ cohomological splitting in the case where $H^{*}(G ; \mathbb{Z})$ has $p$-torsion. It is well known that if $G$ is a compact simply connected Lie group, then $H^{*}(G ; \mathbb{Z})$ is $p$-torsion free if and only if $H^{*}\left(B G ; \mathbb{F}_{p}\right)$ is a polynomial algebra generated by elements with even degrees. Therefore, we pursue the problem for $p=2$ assuming that $G$ is a connected finite loop space and $H^{*}\left(B G ; \mathbb{F}_{2}\right)$ is a polynomial algebra which admits generators of odd degrees. In this case, we find an obstruction for $P \times$ ad $G \rightarrow M$ to split on mod 2 cohomology in the Hochschild homology

$$
\begin{aligned}
H H_{*}\left(H^{*}\left(B G ; \mathbb{F}_{2}\right)\right. & \left., H^{*}\left(M ; \mathbb{F}_{2}\right)\right) \\
& =\operatorname{Tor}_{H^{*}\left(B G ; \mathbb{F}_{2}\right) \otimes H^{*}\left(B G ; \mathbb{F}_{2}\right)}\left(H^{*}\left(B G ; \mathbb{F}_{2}\right), H^{*}\left(M ; \mathbb{F}_{2}\right)\right) .
\end{aligned}
$$

More precisely, we establish the following theorem.

Theorem 1.3. Let $P \rightarrow M$ be a principal $G$-bundle with the classifying map $f: M \rightarrow B G$. Let $\mathfrak{D}: \overline{H^{*}} \rightarrow H H_{*}\left(H^{*}\left(B G ; \mathbb{F}_{2}\right)\right)$ denote the module derivation defined in [12]. Suppose that $H^{*}\left(B G ; \mathbb{F}_{2}\right)$ is a polynomial algebra. Then the following three conditions are equivalent:

(i) the adjoint bundle $P \times{ }_{\mathrm{ad}} G \rightarrow M$ splits on mod 2 cohomology; 
(ii) there exists a normalized system $S$ of generators satisfying the following condition $C(\mathfrak{D}, f)$ : the composition

$$
\begin{aligned}
\left\{\mathrm{Sq}^{\operatorname{deg} y_{i}-1} y_{i}\right\}_{y_{i} \in S} \cap\left(\overline{H^{*}} \cdot \overline{H^{*}}\right) & \stackrel{\mathfrak{D}}{\longrightarrow} H H_{*}\left(H^{*}\left(B G ; \mathbb{F}_{2}\right)\right) \\
& \stackrel{H H_{*}\left(1, f^{*}\right)}{\longrightarrow} H H_{*}\left(H^{*}\left(B G ; \mathbb{F}_{2}\right), H^{*}\left(M ; \mathbb{F}_{2}\right)\right)
\end{aligned}
$$

is the zero map, where $\overline{H^{*}}=\bigoplus_{i>1} H^{i}\left(B G ; \mathbb{F}_{2}\right)$;

(iii) the condition $C(\mathfrak{D}, f)$ holds for any normalized system $S$ of generators.

See Section 2 for the definitions of the module derivation and of the normalized system of generators of $H^{*}\left(B G ; \mathbb{F}_{2}\right)$.

Theorem 1.3 leads us to the following proposition.

Proposition 1.4. Let $n$ be an integer greater than 1. For any $S O(n)$ bundle $P \rightarrow M$ over a connected space $M$, the adjoint bundle $P \times{ }_{\text {ad }} S O(n)$ splits on mod 2 cohomology if and only if the map $H^{*}\left(B S O(n) ; \mathbb{F}_{2}\right) \rightarrow$ $H^{*}\left(M ; \mathbb{F}_{2}\right)$ induced by the classifying map of the bundle $P \rightarrow M$ is trivial.

As mentioned above, if $H^{*}(G ; \mathbb{Z})$ has no $p$-torsion, then all $G$-adjoint bundles admit mod $p$ cohomological splittings without depending on the classifying maps of the corresponding principal $G$-bundles. However, the isomorphisms which give the natural splittings are not compatible with the Steenrod operations in general; even the isomorphisms due to Kono and Kozima [10] and Iwase [7] do not have this property (see Example 3.6 below). The absence of the compatibility enables us to obtain a theorem concerning the cohomology of the classifying space of the loop group.

TheOREM 1.5. Let $p$ be an odd prime and $G$ a connected finite loop space with

$$
H^{*}(G ; \mathbb{Q}) \cong \Lambda\left(x_{2 m_{1}-1}, \ldots, x_{2 m_{l}-1}\right),
$$

where $\operatorname{deg} x_{2 m_{i}-1}=2 m_{i}-1$ and $m_{1} \leq \ldots \leq m_{l}$. Suppose that the least integer of $m_{1}, \ldots, m_{l}$ greater than 1 is prime to $p$, if any. Then the bundle $G \rightarrow B L G \rightarrow B G$ admits an $\mathcal{A}(p)$-cohomological splitting if and only if $H^{*}\left(G ; \mathbb{F}_{p}\right) \cong H^{*}\left(T ; \mathbb{F}_{p}\right)$ as an algebra, where $T$ denotes an appropriate torus.

As an immediate corollary, it follows that if $G$ is simply connected, then a mod $p$ cohomological splitting is not realizable with any map from $B L G$ to $B G \times G$ nor from $B G \times G$ to $B L G$ even though such an algebraic splitting is possible. Observe that the condition on the rational cohomology of $G$ in the above theorem is satisfied when $G$ is a connected Lie group. In this case, we can take 2 as the least integer mentioned in Theorem 1.5.

Some explicit calculation of the Steenrod operations in the mod 2 cohomology of $S U(n)$ and $S p(n)$ allows us to obtain the following theorem. 
THEOREM 1.6. Let $H$ be a compact simply connected simple Lie group. Suppose that a given simply connected finite loop space $G$ is a fake Lie group of type $H$ in the sense of Notbohm and Smith [13]. Then the bundle $G \rightarrow B L G \rightarrow B G$ admits an $\mathcal{A}(p)$-cohomological splitting if and only if $G$ is a fake Lie group of type $S^{3}$ and $p=2$.

It is interesting to mention geometrical facts due to Iwase and Kono [8] and Castellana and Kitchloo [3] which are related to Theorems 1.5 and 1.6. The first one relies on Hubbuck's Torus Theorem. The second has been deduced by applying the localization functor due to Farjoun.

FACT $1([8])$. Let $G$ be a connected finite loop space. There is a homotopy equivalence $\phi: B L G \rightarrow B G \times G$ which satisfies $\phi \circ j \simeq \operatorname{in}_{2}$ if and only if $G \simeq T$.

FACT $2([3])$. For any compact simply connected simple Lie group $G$, $B L G$ is not homotopy equivalent to $B G \times G$ even after completion at any prime $p$.

These facts describe the geometrical rigidity of the classifying space $B L G$, which is not detected exactly at the cohomology algebra level. Our geometrical result below follows from knowledge of the Steenrod operations on $H^{*}\left(B L G ; \mathbb{F}_{p}\right)$ which we gain in the proof of Theorem 1.5.

COROLlary 1.7. If $G$ is a simply connected finite loop space, then for any non-negative integer $n$, there is no homotopy equivalence $\phi: \Sigma^{n} B L G \rightarrow$ $\Sigma^{n}(B G \times G)$ such that $\phi \circ \Sigma^{n} j \simeq \Sigma^{n} \mathrm{in}_{2}$.

By analyzing the action of the Steenrod operation $\wp^{1}$ on the algebra $H^{*}\left(P \times{ }_{\text {ad }} S U(n) ; \mathbb{F}_{p}\right)$ with the Wu formula due to Shay [14], we obtain another geometrical result. It asserts that, under an appropriate assumption on $M$ and the integer $n$, the isomorphism type of an $S U(n)$-adjoint bundle over $M$ coincides with the homotopy type of the bundle in the sense mentioned before Definition 1.1.

THEOREM 1.8. Let $M$ be a 4- or 5-dimensional $C W$ complex for which $H^{4}(M ; \mathbb{Z})=\mathbb{Z} / p$, where $p$ is odd prime. Then the natural surjection

$\mathbb{Z} / p \approx\{S U(n)$-adjoint bundles over $M\} / \cong$

$$
\rightarrow\{S U(n) \text {-adjoint bundles over } M\} / \simeq
$$

from the set of isomorphism classes of $S U(n)$-adjoint bundles over $M$ to that of homotopy equivalence classes of the bundles is bijective if $n=p$ or $p-1$.

We now briefly describe the organization of this paper. In Section 2, we recall the definition of a module derivation and the Koszul-Tate resolution 
for explicit calculation of an Eilenberg-Moore spectral sequence. In Section 3, after proving Theorem 1.2, we study the Steenrod operations on the mod $p$ cohomology of $P \times{ }_{\text {ad }} G$ using a module derivation. Section 4 is devoted to proving Theorems 1.3, 1.5 and 1.6 and Proposition 1.4. In Section 5, we prove Theorem 1.8 after a comment on the classification of $G$-adjoint bundles under rational homotopy equivalence in the sense of Halperin and Thomas [6]. Our further expectations concerning module derivations are described in the last section.

2. Preliminaries. In order to determine the algebra structure of $H^{*}\left(P \times_{\mathrm{ad}} G ; \mathbb{F}_{p}\right)$ over $\mathcal{A}(p)$, we shall calculate explicitly the EilenbergMoore spectral sequence, which is constructed in Section 3. To this end, we need the Koszul-Tate resolution and a morphism between this resolution and the bar resolution.

Lemma 2.1 ([17, Proposition 3.5], [11, Propositions 1.1, 1.5]). Let $A$ be a polynomial algebra over $\mathbb{F}_{p}$, say $A=\mathbb{F}_{p}\left[y_{1}, \ldots, y_{n}\right]$.

(i) There exists a projective resolution $\mathcal{K} \bullet \stackrel{\varphi}{\rightarrow} A \rightarrow 0$ of $A$ as a left $A \otimes A$-module such that

$$
\mathcal{K}^{\bullet}=A \otimes A \otimes \Lambda\left(\bar{y}_{1}, \ldots, \bar{y}_{l}\right),
$$

$d\left(\bar{y}_{i}\right)=y_{i} \otimes 1-1 \otimes y_{i}$ and $\varphi$ is the multiplication of $A$, where $\operatorname{bideg} \bar{y}_{i}=$ $\left(-1, \operatorname{deg} y_{i}\right)$.

(ii) Let $B^{\bullet}(A \otimes A, A) \rightarrow A \rightarrow 0$ be the bar resolution of $A$ as a left $A \otimes A$-module. Then there exists a morphism of resolutions $\Psi^{\sharp}=\left\{\psi_{-n}^{\sharp}\right\}$ : $B^{\bullet}(A \otimes A, A) \rightarrow \mathcal{K}^{\bullet}$ such that $\psi_{0}^{\sharp}(a \otimes b \otimes c)=a \otimes b c$ and $\psi_{-1}^{\sharp}\left(1 \otimes 1\left[y_{i} \otimes 1\right.\right.$ $\left.\left.-1 \otimes y_{i}\right] 1\right)=\bar{y}_{i}$. Hence, for any graded commutative right A-algebra $N$, $\Psi^{\sharp}$ induces an isomorphism of algebras

$$
\Psi: \operatorname{Tor}_{A \otimes A}(N, A)_{B} \cong \operatorname{Tor}_{A \otimes A}(N, A)_{\mathrm{KT}}
$$

for which $\Psi\left(1_{N}\left[y_{i} \otimes 1-1 \otimes y_{i}\right] 1_{A}\right)=\bar{y}_{i}$, where $\operatorname{Tor}_{A \otimes A}(N, A)_{B}$ and $\operatorname{Tor}_{A \otimes A}(N, A)_{\mathrm{KT}}$ denote the torsion products obtained from the bar resolution and the Koszul-Tate resolution respectively, and the $A \otimes A$-module structure on $N$ is defined by composing the multiplication of $A$ with the $A$-module structure of $N$.

The Steenrod operations in the Eilenberg-Moore spectral sequence can be expressed in terms of the bar complex which computes the $E_{2}$-term (see [16]). Therefore Lemma 2.1 plays a crucial role in our computation using the Eilenberg-Moore spectral sequence.

Following [12], we recall the general definition of a module derivation.

Definition 2.2. Let $A$ be a graded commutative algebra over $\mathbb{F}_{p}$ and $L$ a left $A$-module. A module derivation of $A$ with values in $L$ is an $\mathbb{F}_{p}$-linear 
map $\mathfrak{D}: A \rightarrow L$ of degree -1 such that

$$
\mathfrak{D}(a b)=(-1)^{(\operatorname{deg} a+1) \operatorname{deg} b} b \mathfrak{D}(a)+(-1)^{\operatorname{deg} a} a \mathfrak{D}(b)
$$

for any $a$ and $b$ in $A$.

Observe that module derivations satisfy the uniqueness condition in the sense that, for module derivations $\mathfrak{D}_{1}$ and $\mathfrak{D}_{2}$ from $A$ to $L$, if $\mathfrak{D}_{1}\left(x_{i}\right)=$ $\mathfrak{D}_{2}\left(x_{i}\right)$ for algebra generators $x_{i}$ of $A$, then $\mathfrak{D}_{1}=\mathfrak{D}_{2}$ on $A$.

Let $N$ be a graded commutative $A$-bialgebra over $\mathbb{F}_{p}$. We now define an $\mathbb{F}_{p}$-linear map

$\mathfrak{D}_{N}: A \rightarrow H H(A, N)=\operatorname{Tor}_{A \otimes A}(N, A)=H\left(N \otimes_{A \otimes A} B^{\bullet}(A \otimes A, A), 1 \otimes \partial\right)$

by $x \mapsto 1_{N}[x \otimes 1-1 \otimes x] 1_{A}$. An explicit calculation of the differential of the bar complex shows that $\mathfrak{D}_{N}$ is a module derivation with values in the Hochschild homology $H H(A, N)$ ([12, Proposition 3.2]). To simplify, we denote the module derivation $\mathfrak{D}_{N}$ by $\mathfrak{D}$ if $N=A$. In particular, putting $A=$ $H^{*}\left(B G ; \mathbb{F}_{2}\right)$, we obtain the module derivation mentioned in Theorem 1.3.

Let $A$ be a polynomial algebra $\mathbb{F}_{p}\left[y_{1}, \ldots, y_{l}\right]$. Observe that generators with odd degrees are allowed in $A$ if $p=2$. By Lemma 2.1(ii), we can identify the Hochschild homology of $A$ given by the bar complex with $\operatorname{Tor}_{A \otimes A}(A, A)_{\mathrm{KT}}$. Thus the module derivation $\mathfrak{D}$ is regarded as the map

$$
\mathfrak{D}: A=\mathbb{F}_{p}\left[y_{1}, \ldots, y_{l}\right] \rightarrow H H(A, A)=\mathbb{F}_{p}\left[y_{1}, \ldots, y_{l}\right] \otimes \Lambda\left(\bar{y}_{1}, \ldots, \bar{y}_{l}\right)
$$

defined by $y_{i} \mapsto \bar{y}_{i}$.

We now introduce the notion a normalized system of generators of $H^{*}\left(B G ; \mathbb{F}_{2}\right)$ and show the existence of such systems. Let $A$ be a polynomial algebra over the Steenrod algebra $\mathcal{A}(2)$ with finitely many generators.

Definition 2.3. A set $S=\left\{y_{i}\right\}_{1 \leq i \leq l}$ of indecomposable elements of $A$ is said to be a normalized system of generators if:

(1) $A \cong \mathbb{F}_{2}\left[y_{1}, \ldots, y_{l}\right]$ as an algebra,

(2) if $\mathrm{Sq}^{\operatorname{deg} y_{i}-1} y_{i}$ is an indecomposable element, then $\mathrm{Sq}^{\operatorname{deg} y_{i}-1} y_{i} \in S$.

For the rest of this paper, we denote by $\mathrm{Sq}^{*}: A \rightarrow A$ the operation defined by $\mathrm{Sq}^{*} x=\mathrm{Sq}^{\operatorname{deg} x-1} x$. Notice that the set $S \cup\{0\}$ is not necessarily invariant under the action of $\mathrm{Sq}^{*}$.

Lemma 2.4. Every polynomial algebra $A$ over $\mathcal{A}(2)$ has a normalized system of generators.

Proof. We argue by induction on the degree of $A$. Let $k$ be the least degree such that $A^{k} \neq 0$ and $k>0$. We begin the induction by letting $S_{k}$ be a basis of $(Q A)^{k}$. Suppose that there exists a set $S_{l}$ consisting of indecomposable elements of $A$ such that conditions (1) and (2) are satisfied below degree $l$. Then we can write

$$
(Q A)^{(l+2) / 2}=\left\langle y_{i}\right\rangle_{i \in J_{0}} \oplus \ldots \oplus\left\langle y_{i}\right\rangle_{i \in J_{s}} .
$$


Here $\left\langle y_{i}\right\rangle_{i \in J_{r}}$ denotes the vector space spanned by the elements $y_{i}$ for $i \in J_{r}$ such that

$$
y_{i}=\underbrace{\left(\mathrm{Sq}^{*} \circ \ldots \circ \mathrm{Sq}^{*}\right)}_{r \text { times }} y_{k}=:\left(\mathrm{Sq}^{*}\right)^{r} y_{k}
$$

for some $y_{k} \in S_{l}$ and $y_{i}$ is not in the image of the $(r+1)$-fold composition $\left(\mathrm{Sq}^{*}\right)^{r+1}$. In what follows, if $y_{i}=\left(\mathrm{Sq}^{*}\right)^{s} y$ for some $y \in S_{l}$, then we denote by $\left(\mathrm{Sq}^{*}\right)^{-s_{0}} y_{i}$ the element $\left(\mathrm{Sq}^{*}\right)^{s-s_{0}} y$.

Choose a subset $J_{s}^{\prime}$ of $J_{s}$ so that $\mathrm{Sq}^{*} y_{i}\left(i \in J_{s}^{\prime}\right)$ are linearly independent in the vector space $\left\langle\mathrm{Sq}^{*} y_{i}\right\rangle_{i \in J_{s}}$. For any $j \in J_{s} \backslash J_{s}^{\prime}$, we can write $\mathrm{Sq}^{*} y_{j}=$ $\sum_{i \in J_{s}^{\prime}} \alpha_{i} \mathrm{Sq}^{*} y_{i}$ for some $\alpha_{i} \in \mathbb{F}_{2}$. Replace $\left(\mathrm{Sq}^{*}\right)^{-s_{0}} y_{j}$ with

$$
\left(\mathrm{Sq}^{*}\right)^{-s_{0}} y_{j}-\sum_{i \in J_{s}^{\prime}} \alpha_{i}\left(S q^{*}\right)^{-s_{0}} y_{i}
$$

for any $s_{0}\left(0 \leq s_{0} \leq s\right)$. After the replacement, it is readily seen that $\left(\mathrm{Sq}^{*}\right) y_{j}$ is decomposable for any $j \in J_{s} \backslash J_{s}^{\prime}$ and that the new set $S_{l}$ also satisfies (1) and (2) below degree $l$. By extending $\left\{\mathrm{Sq}^{*} y_{i}\right\}_{i \in J_{s}^{\prime}}$, we choose a basis of $\left\langle\mathrm{Sq}^{*} y_{i}\right\rangle_{i \in J_{s-1} \cup J_{s}^{\prime}}$, say $\left\{\mathrm{Sq}^{*} y_{i}\right\}_{i \in J_{s-1}^{\prime}} \cup\left\{\mathrm{Sq}^{*} y_{i}\right\}_{i \in J_{s}^{\prime}}$. The same argument applies to replacing $\left(\mathrm{Sq}^{*}\right)^{-t} y_{j}$ for $j \in J_{s-1} \backslash J_{s-1}^{\prime}$ and $0 \leq t \leq s-1$. Thus we obtain a new set $S_{l}$ such that $\left(\mathrm{Sq}^{*}\right) y_{j}$ is decomposable for any $j \in J_{s-1} \backslash J_{s-1}^{\prime}$ and satisfies (1) and (2) below degree $l$. In consequence, by choosing appropriate elements $y_{i} \in(Q A)^{q+1}\left(i \in I_{0}\right)$, we can write

$$
(Q A)^{l+1}=\left\langle y_{i}\right\rangle_{i \in I_{0}} \oplus\left\langle\mathrm{Sq}^{*} y_{i}\right\rangle_{i \in J_{0}^{\prime}} \oplus \ldots \oplus\left\langle\mathrm{Sq}^{*} y_{i}\right\rangle_{i \in J_{s}^{\prime}}
$$

Put $S_{l+1}=S_{l} \cup\left\{y_{i}\right\}_{i \in I_{0}} \cup\left\{\mathrm{Sq}^{*} y_{i}\right\}_{i \in J_{0}^{\prime} \cup \ldots \cup J_{s}^{\prime}}$. Then $S_{l+1}$ satisfies (1) and (2) below degree $l+1$.

\section{Proof of Theorem 1.2 and the $\mathcal{A}(p)$-action on the cohomology} of $P \times$ ad $G$. In the proof of Theorem 1.2, we use an Eilenberg-Moore spectral sequence converging to $H^{*}\left(P \times_{\text {ad }} G ; \mathbb{F}_{p}\right)$. In order to obtain the spectral sequence in the usual manner, we first construct an appropriate homotopy fibre square.

Let $H$ be a closed subgroup of $G^{2}=G \times G$ which acts from the left on $G$ by $(k, h) \cdot g=k g h^{-1}$. Let $\delta G$ denote the closed subgroup of $G^{2}$ defined by $\delta G=\left\{(g, g) \in G^{2} \mid g \in G\right\}$. In [4, Section 3], Eschenburg has introduced a bundle map

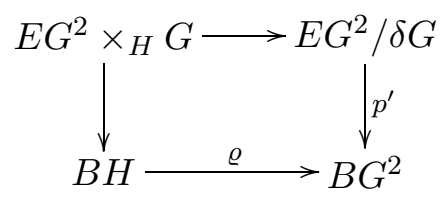

which is a homeomorphism on the fibres. Here $\varrho: B H \rightarrow B G^{2}$ is induced by the inclusion $H \rightarrow G^{2}$. 
The diagonal homomorphism $\Delta: G \rightarrow G \times G$ induces the maps $E \Delta$ : $E G \rightarrow E G^{2}$ and $\eta=B \Delta: B G \rightarrow E G^{2} / \delta G$ which are homotopy equivalences. Moreover, since there exists a morphism of fibrations:

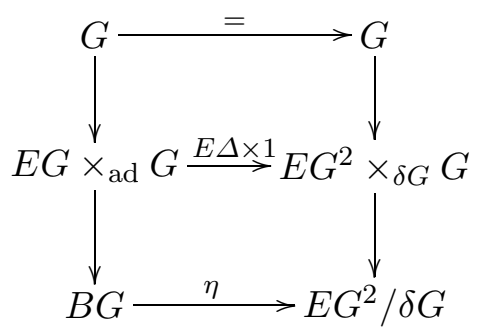

it follows that the spaces $E G \times{ }_{\text {ad }} G$ and $E G^{2} \times{ }_{\delta G} G$ are of the same homotopy type. By taking $H=\delta G$ and by combining the above fibre square with (3.1), we obtain a fibre square

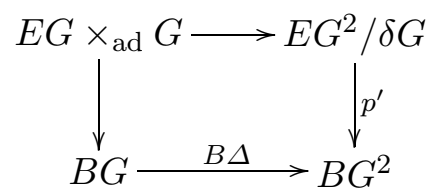

Let $f: M \rightarrow B G$ be the classifying map of a given $G$-bundle $P \rightarrow M$. Then the map $f$ fits in the diagram of fibre squares

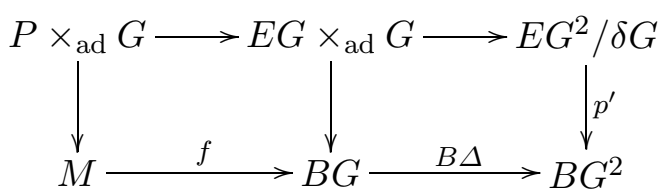

Moreover we have a commutative diagram

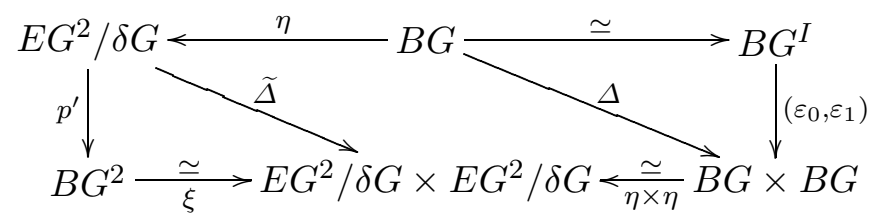

in which $\varepsilon_{i}(\gamma)=\gamma(i)$ for $\gamma \in B G^{I}$, the map $\xi$ is a homotopy equivalence defined by $\xi\left(\left(x_{1}, y_{1}\right) t \oplus \ldots\right)=\left(\left(x_{1}, x_{1}\right) t \oplus \ldots,\left(y_{1}, y_{1}\right) t \oplus \ldots\right)$ and $\widetilde{\Delta}=\xi p^{\prime}$. Combining the diagrams (3.3) and (3.4), we get a morphism of homotopy 
fibre squares

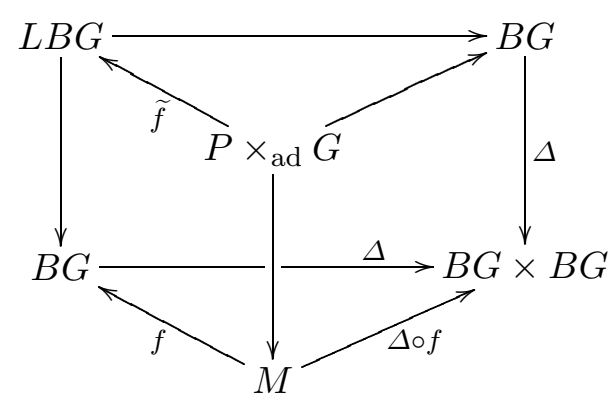

where $L B G$ denotes the space of free loops on $B G$. Observe that $\xi \circ B \Delta \circ f=$ $(\eta \times \eta) \circ(\Delta \circ f)$. The right front square enables us to obtain the EilenbergMoore spectral sequence $\left\{E_{r}^{*, *}, d_{r}\right\}$ converging to $H^{*}\left(P \times_{\text {ad }} G ; \mathbb{F}_{p}\right)$ with

$$
E_{2}^{*, *} \cong \operatorname{Tor}_{H^{*}\left(B G ; \mathbb{F}_{p}\right) \otimes H^{*}\left(B G ; \mathbb{F}_{p}\right)}^{* *}\left(H^{*}\left(M ; \mathbb{F}_{p}\right), H^{*}\left(B G ; \mathbb{F}_{p}\right)\right)
$$

as an algebra. Let $F^{*} H^{*}$ denote the filtration of $H^{*}\left(P \times_{\text {ad }} G ; \mathbb{F}_{p}\right)$ which is given by the Eilenberg-Moore spectral sequence $\left\{E_{r}^{*, *}, d_{r}\right\}$ and let $q$ : $F^{-1} H^{*} \rightarrow E_{0}^{-1, *}=F^{-1} H^{*} / F^{0} H^{*}$ be the projection, unless otherwise stated.

Proof of Theorem 1.2. Suppose that $H^{*}(G ; \mathbb{Z})$ is $p$-torsion free. Then $H^{*}\left(B G ; \mathbb{F}_{p}\right)$ is a polynomial algebra with generators in even degrees, say $H^{*}\left(B G ; \mathbb{F}_{p}\right) \cong \mathbb{F}_{p}\left[y_{1}, \ldots, y_{l}\right]$. By using the Koszul-Tate resolution $\mathcal{K} \bullet \stackrel{\varphi}{\rightarrow}$ $H^{*}\left(B G ; \mathbb{F}_{p}\right) \rightarrow 0$ of $H^{*}\left(B G ; \mathbb{F}_{p}\right)$ as an $H^{*}\left(B G ; \mathbb{F}_{p}\right) \otimes H^{*}\left(B G ; \mathbb{F}_{p}\right)$-module (Lemma 2.1(i)), we see that, as an algebra,

$$
\begin{aligned}
E_{2}^{*, *} & \cong H\left(H^{*}\left(M ; \mathbb{F}_{p}\right) \otimes_{H^{*}\left(B G ; \mathbb{F}_{p}\right) \otimes H^{*}\left(B G ; \mathbb{F}_{p}\right)} \mathcal{K}^{\bullet} ; 1 \otimes d\right) \\
& \cong H\left(H^{*}\left(M ; \mathbb{F}_{p}\right) \otimes \Lambda\left(\bar{y}_{1}, \ldots, \bar{y}_{l}\right) ; d\left(\bar{y}_{i}\right)=(\Delta f)^{*}\left(y_{i} \otimes 1-1 \otimes y_{i}\right)\right),
\end{aligned}
$$

where $\operatorname{bideg} x=(0, \operatorname{deg} x)$ for $x \in H^{*}\left(M ; \mathbb{F}_{p}\right)$ and $\operatorname{bideg} \bar{y}_{i}=\left(-1, \operatorname{deg} y_{i}\right)$. Since the differential $d$ of this complex is trivial, it follows that $E_{2}^{*, *} \cong$ $H^{*}\left(M ; \mathbb{F}_{p}\right) \otimes \Lambda\left(\bar{y}_{1}, \ldots, \bar{y}_{l}\right)$ and $E_{2}^{*, *} \cong E_{\infty}^{*, *}$ for dimensional reasons. It is immediate that, for $p \neq 2$, there is no extension problem in the $E_{\infty}^{*, *}$-term. Thus we have an isomorphism of algebras $H^{*}\left(P \times_{\text {ad }} G ; \mathbb{F}_{p}\right) \cong H^{*}\left(M ; \mathbb{F}_{p}\right) \otimes$ $\Lambda\left(\bar{y}_{1}, \ldots, \bar{y}_{l}\right)$.

Observe that the elements $\bar{y}_{i}(i=1, \ldots, l)$ in $H^{*}\left(P \times_{\text {ad }} G ; \mathbb{F}_{p}\right)$ can be chosen so that $s^{*}\left(\bar{y}_{i}\right)=0$, where $s: M \rightarrow P \times \times_{\text {ad }} G$ is a section of the projection $\pi: P \times_{\text {ad }} G \rightarrow M$. In the case $p=2$, we must solve extension problems that whether $\bar{y}_{i}^{2}=0$ or not. Since $\bar{y}_{i}^{2}=0$ in $E_{\infty}^{-2, *}$, it follows that $\bar{y}_{i}^{2}$ belongs to $F^{-1} H^{*}$. Moreover, using the Steenrod operation in the spectral sequence [16], we see that $\bar{y}_{i}^{2}=\operatorname{Sq}^{\operatorname{deg} y_{1}-1} \bar{y}_{i}=\operatorname{Sq}^{\operatorname{deg} y_{1}-1}\left(1\left[y_{i} \otimes 1-1 \otimes y_{i}\right] 1\right)=$ $1\left[\mathrm{Sq}^{\operatorname{deg} y_{1}-1} y_{i} \otimes 1-1 \otimes \mathrm{Sq}^{\operatorname{deg} y_{1}-1} y_{i}\right] 1=0$ in $F^{-1}$. Thus $\bar{y}_{i}^{2}=\pi^{*} Q$ for some $Q \in H^{*}\left(M ; \mathbb{F}_{p}\right)$. It is now readily seen that $0=s^{*}\left(\bar{y}_{i}^{2}\right)=Q$. 
In order to define an isomorphism which gives a mod $p$ cohomological splitting, consider the morphism of homotopy fibre squares

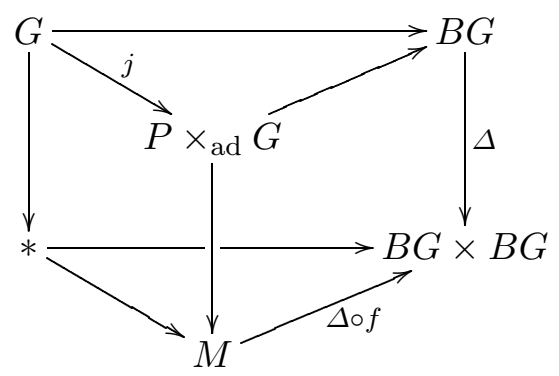

Let $\left\{\widehat{E}_{r}^{* * *}, \widehat{d}_{r}\right\}$ be the Eilenberg-Moore spectral sequence for the back fibre square in the above diagram. By making use of the Koszul-Tate resolution, we see that, as algebras,

$$
\begin{aligned}
\widehat{E}_{2}^{*, *} & \cong \operatorname{Tor}_{H^{*}\left(B G ; \mathbb{F}_{p}\right) \otimes H^{*}\left(B G ; \mathbb{F}_{p}\right)}\left(\mathbb{F}_{p}, H^{*}\left(B G ; \mathbb{F}_{p}\right)\right) \\
& \cong H\left(\mathbb{F}_{p} \otimes_{H^{*}\left(B G ; \mathbb{F}_{p}\right) \otimes H^{*}\left(B G ; \mathbb{F}_{p}\right)} \mathcal{K}^{\bullet}, 1 \otimes d\right) \cong \Lambda\left(\widetilde{y}_{1}, \ldots, \widetilde{y}_{l}\right) .
\end{aligned}
$$

For dimensional reasons, it follows that $\widehat{E}_{2}^{* * *} \cong \widehat{E}_{\infty}^{*, *} \cong \widehat{E}_{0}^{*, *}$ as bigraded algebras. Comparing $\left\{E_{r}^{*, *}, d_{r}\right\}$ and $\left\{\widehat{E}_{r}^{*, *}, \widehat{d}_{r}\right\}$ with the morphism of spectral sequences induced by the morphism of the fibre squares, we obtain a morphism of bigraded algebras $E_{0}(j): E_{0}^{*, *} \rightarrow \widehat{E}_{0}^{*, *}=\Lambda\left(\widetilde{y}_{1}, \ldots, \widetilde{y}_{l}\right)$ such that $E_{0}(j)\left(\bar{y}_{i}\right)=\widetilde{y}_{i}$ and the restriction of $E_{0}(j)$ to $E_{0}^{0, *}$ is the trivial map. Define an isomorphism $\phi: H^{*}\left(P \times_{\text {ad }} G ; \mathbb{F}_{p}\right) \rightarrow H^{*}\left(M ; \mathbb{F}_{p}\right) \otimes H^{*}\left(G ; \mathbb{F}_{p}\right)$ of $H^{*}\left(M ; \mathbb{F}_{p}\right)$-algebras by $\phi\left(\bar{y}_{i}\right)=\widetilde{y}_{i}$. Since $H^{*}\left(G ; \mathbb{F}_{p}\right)$ is regarded as the total complex Total $\widehat{E}_{0}^{*, *}$, it follows that $\operatorname{in}_{2}^{*} \circ \phi=j^{*}$.

As mentioned in the introduction, the natural isomorphism of $H^{*}\left(M ; \mathbb{F}_{p}\right)$ algebras in Theorem 1.2 is not compatible with the Steenrod operations in general. In the rest of this section, we show that this follows from some explicit calculation of the Steenrod operations on $H^{*}\left(P \times_{\text {ad }} G ; \mathbb{F}_{p}\right)$ and that the module derivation $\mathfrak{D}_{H^{*}\left(M ; \mathbb{F}_{p}\right)}: H^{*}\left(B G ; \mathbb{F}_{p}\right) \rightarrow H H_{*}\left(H^{*}\left(B G ; \mathbb{F}_{p}\right)\right.$, $\left.H^{*}\left(M ; \mathbb{F}_{p}\right)\right)$ plays an important role in the calculation. We remark that the $H^{*}\left(B G ; \mathbb{F}_{p}\right)$-bimodule structure of $H^{*}\left(M ; \mathbb{F}_{p}\right)$ is defined naturally via the homomorphism $f^{*}$ and multiplication on $H^{*}\left(M ; \mathbb{F}_{p}\right)$, where $f: M \rightarrow B G$ is the classifying map of the given $G$-bundle $P \rightarrow M$.

We consider the $\mathcal{A}(p)$-algebra structure of $H^{*}\left(P \times_{\text {ad }} G ; \mathbb{F}_{p}\right)$ assuming that $H^{*}\left(B G ; \mathbb{F}_{p}\right)$ is a polynomial algebra $\mathbb{F}_{p}\left[y_{1}, \ldots, y_{l}\right]$. As for the case $p=2$, the polynomial algebra is not necessarily evenly generated.

Consider the Eilenberg-Moore spectral sequence $\left\{E_{r}^{*, *}, d_{r}\right\}$ converging to $H^{*}\left(P \times_{\mathrm{ad}} G ; \mathbb{F}_{2}\right)$. As in the proof of Theorem 1.2, the spectral sequence 
collapses at the $E_{2}$-term and hence

$$
H^{*}\left(P \times_{\text {ad }} G ; \mathbb{F}_{2}\right) \cong H^{*}\left(M ; \mathbb{F}_{2}\right) \otimes \Delta\left(\bar{y}_{1}, \ldots, \bar{y}_{l}\right)
$$

as an $H^{*}\left(M ; \mathbb{F}_{2}\right)$-algebra, where $\Delta\left(\bar{y}_{1}, \ldots, \bar{y}_{l}\right)$ denotes the algebra generated by a 2 -simple system of generators $\bar{y}_{1}, \ldots, \bar{y}_{l}$. In the case $p \neq 2$, we recall the $H^{*}\left(M ; \mathbb{F}_{p}\right)$-algebra structure of $H^{*}\left(P \times_{\text {ad }} G ; \mathbb{F}_{p}\right)$ from the proof of Theorem 1.2:

$$
H^{*}\left(P \times_{\text {ad }} G ; \mathbb{F}_{p}\right) \cong H^{*}\left(M ; \mathbb{F}_{p}\right) \otimes \Lambda\left(\bar{y}_{1}, \ldots, \bar{y}_{l}\right) .
$$

Theorem 3.1 below asserts that the action of the Steenrod operations on $H^{*}\left(P \times_{\text {ad }} G ; \mathbb{F}_{p}\right)$ is determined explicitly by the actions on $H^{*}\left(B G ; \mathbb{F}_{p}\right)$ and $H^{*}\left(M ; \mathbb{F}_{p}\right)$ via the induced map $f^{*}: H^{*}\left(B G ; \mathbb{F}_{p}\right) \rightarrow H^{*}\left(M ; \mathbb{F}_{p}\right)$ and a module derivation with values in $H^{*}\left(P \times_{\text {ad }} G ; \mathbb{F}_{p}\right)$.

TheOREM 3.1. There exists a module derivation

$$
\mathfrak{D}_{f}^{\prime}: H^{*}\left(B G ; \mathbb{F}_{p}\right) \rightarrow H^{*-1}\left(P \times_{\text {ad }} G ; \mathbb{F}_{p}\right)
$$

which is compatible with the action of the Steenrod operations. In particular, $\mathrm{Sq}^{i} \bar{y}_{j}=\mathfrak{D}_{f}^{\prime} \mathrm{Sq}^{i} y_{j}$ and $\wp^{i} \bar{y}_{j}=\mathfrak{D}_{f}^{\prime} \wp^{i} y_{j}, \beta \wp^{i} \bar{y}_{j}=0$ if $p \neq 2$.

In what follows, we denote by $\mathfrak{D}^{\prime}$ the module derivation $\mathfrak{D}_{\mathrm{id}}^{\prime}: H^{*}\left(B G ; \mathbb{F}_{p}\right)$ $\rightarrow H^{*-1}\left(B L G ; \mathbb{F}_{p}\right)$.

Let $X$ be a simply connected space and $L X$ the free loop space. In order to prove Theorem 3.1, we first consider the Eilenberg-Moore spectral sequence $\left\{E_{r}, d_{r}\right\}$ of the homotopy fibre square

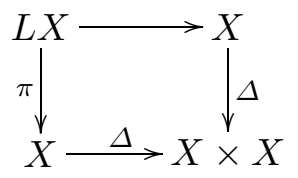

where $\Delta$ denotes the diagonal map and $\pi: L X \rightarrow X$ is the projection defined by $\pi(\gamma)=\gamma(0)$ for $\gamma \in L X$. Let $r: E_{2}^{-1, *} \rightarrow E_{\infty}^{-1, *}$ be the composition of the projections $p_{r}: E_{r}^{-1, *} \rightarrow E_{r+1}^{-1, *}$ and let $q: F^{-1} H^{*}\left(L X ; \mathbb{F}_{p}\right) \rightarrow E_{\infty}^{-1, *}$ denote the projection defined by using the filtration $\left\{F^{-i} H^{*}\left(L X ; \mathbb{F}_{p}\right)\right\}$ of $H^{*}\left(L X ; \mathbb{F}_{p}\right)$. The following theorem enables us to realize the module derivation $\mathfrak{D}: H^{*}\left(X ; \mathbb{F}_{p}\right) \rightarrow H H\left(H^{*}\left(X ; \mathbb{F}_{p}\right), H^{*}\left(X ; \mathbb{F}_{p}\right)\right)$, which is defined algebraically in Section 2, by means of a continuous map via the spectral sequence $\left\{E_{r}, d_{r}\right\}$.

Theorem 3.2 ([12, Propositions 3.3 and 3.4]). Let ev $: S^{1} \times L X \rightarrow X$ be the evaluation map and $\int_{S^{1}}: H^{*}\left(S^{1} \times L X ; \mathbb{F}_{p}\right) \rightarrow H^{*-1}\left(L X ; \mathbb{F}_{p}\right)$ be integration along $S^{1}$.

(i) The composition $\mathfrak{D}_{X}=\int_{S^{1}} \circ \mathrm{ev}^{*}$ is a module derivation with values in $F^{-1} H^{*}\left(L X ; \mathbb{F}_{p}\right)$. 
(ii) The composition $r \circ \mathfrak{D}: H^{*}\left(X ; \mathbb{F}_{p}\right) \rightarrow H H^{-1, *}(A, A) \cong E_{2}^{-1, *} \rightarrow$ $E_{\infty}^{-1, *}$ coincides with $q \circ \mathfrak{D}_{X}: H^{*}\left(X ; \mathbb{F}_{p}\right) \rightarrow F^{-1} H^{*-1}\left(L X ; \mathbb{F}_{p}\right) \rightarrow E_{\infty}^{-1, *}$.

We also need the following lemma to prove Theorem 3.1.

LEMMA 3.3. Let $s_{X}$ be a section of the projection $\pi: L X \rightarrow X$. Then $s_{X}^{*} \circ \mathfrak{D}_{X}=0$.

Proof. For any $y \in H^{l}(X ; \mathbb{Z} / p)$, we can choose a map $X \rightarrow K=$ $K(\mathbb{Z} / p ; l)$ which represents $y$. It is immediate that $s_{K}^{*} \mathfrak{D}_{K}=0$ in $H^{l}\left(K ; \mathbb{F}_{p}\right)$ for dimensional reasons. From the naturality of the module derivation $\mathfrak{D}_{X}$, we have the result.

Proof of Theorem 3.1. Let $s: M \rightarrow P \times{ }_{\text {ad }} G$ be a section of the projection $P \times_{\text {ad }} G \rightarrow M$. We can choose $\bar{y}_{i} \in H^{*}\left(P \times_{\text {ad }} G ; \mathbb{F}_{p}\right)(i=1, \ldots, l)$ so that $s^{*}\left(\bar{y}_{i}\right)=0$ and $\bar{y}_{i} \in F^{-1} H^{*}$. We define a module derivation $\mathfrak{D}_{f}^{\prime}$ : $H^{*}\left(B G ; \mathbb{F}_{p}\right) \rightarrow F^{-1} H^{*}$ by $\mathfrak{D}_{f}^{\prime}\left(y_{i}\right)=\bar{y}_{i}$. Apply the Eilenberg-Moore spectral sequence to the diagram (3.5). Then the naturality of the spectral sequences implies that $\tilde{f}^{*} \mathfrak{D}_{\mathrm{id}}^{\prime}=\mathfrak{D}_{f}^{\prime}$. Here we identify the space $E G \times_{\text {ad }} G$ with the free loop space $L B G$.

To complete the proof, it suffices to show that $\mathfrak{D}_{\text {id }}^{\prime}$ is compatible with the action of the Steenrod operations. Thanks to Theorem $3.2, \mathfrak{D}_{\text {id }}^{\prime}=q \mathfrak{D}_{B G}$ as a map from $H^{*}\left(B G ; \mathbb{F}_{p}\right)$ to $E_{\infty}^{-1, *}$. Thus it is readily seen that, for any $y \in$ $H^{*}\left(B G ; \mathbb{F}_{p}\right)$, there exists $Q \in H^{*-1}\left(B G ; \mathbb{F}_{p}\right)$ such that $\mathfrak{D}_{\mathrm{id}}^{\prime} y=\mathfrak{D}_{B G} y+\pi^{*} Q$. By Lemma 3.3, we have $0=0+s_{B G}^{*} \pi^{*} Q$ and hence $Q=0$. It is immediate that the map $\mathfrak{D}_{B G}$ is compatible with the action of the Steenrod operations. This completes the proof.

REMARK 3.4. Under the notation in the proof of Theorem 3.1, we have $\bar{y}_{j}^{2}=\mathrm{Sq}^{\operatorname{deg} y_{j}-1} \bar{y}_{j}=\mathfrak{D}_{f}^{\prime} \mathrm{Sq}^{\operatorname{deg} y_{i}-1} y_{j}$ in $H^{*}\left(P \times_{\mathrm{ad}} G ; \mathbb{F}_{2}\right)$. This equality enables us to solve extension problems in the $E_{\infty}$-term. In consequence we have

$$
\begin{aligned}
& H^{*}\left(P \times_{\mathrm{ad}} G ; \mathbb{F}_{2}\right) \\
& \quad \cong H^{*}\left(M ; \mathbb{F}_{2}\right) \otimes \mathbb{F}_{2}\left[\bar{y}_{1}, \ldots, \bar{y}_{l}\right] /\left(\bar{y}_{i}^{2}+\mathfrak{D}_{f}^{\prime} \mathrm{Sq}^{\operatorname{deg} y_{i}-1} y_{i} ; i=1, \ldots, l\right)
\end{aligned}
$$

as an $H^{*}\left(M ; \mathbb{F}_{2}\right)$-algebra, where $\operatorname{deg} \bar{y}_{i}=\operatorname{deg} y_{i}-1$.

REMARK 3.5. Let $X$ be a simply connected space whose mod $p$ cohomology is a polynomial algebra. Consider the Eilenberg-Moore spectral sequence $\left\{\widetilde{E}_{r}, \widetilde{d}_{r}\right\}$ for the homotopy fibre square (3.7). Then the same argument as in the proof of Theorem 1.2 yields the algebra structure of $H^{*}\left(L X ; \mathbb{F}_{p}\right)$. Moreover, by applying Theorem 3.2 , we can construct a module derivation $\mathfrak{D}: H^{*}\left(X ; \mathbb{F}_{p}\right) \rightarrow H^{*}\left(L X ; \mathbb{F}_{p}\right)$ which is compatible with the Steenrod operations, as in Theorem 3.1. In consequence the $\mathcal{A}(p)$-algebra 
structure of $H^{*}\left(L X ; \mathbb{F}_{p}\right)$ can be determined from that of $H^{*}\left(X ; \mathbb{F}_{p}\right)$ via the module derivation $\mathfrak{D}$ (see Example 3.6 below).

In the case $p=2$, Bökstedt and Ottosen [1] have also detrermined such an algebra structure over $\mathcal{A}(2)$. We also mention that, by applying Theorem 3.1 , one can recover the result of [3, Theorem 5.1], in which the mod 2 cohomology algebra over $\mathcal{A}(2)$ of $B L G_{2}$ is determined explicitly, with a more systematic manner. Here $G_{2}$ is the compact simply connected simple exceptional Lie group of rank 2 .

EXAMPLE 3.6. Let $c_{i}$ be the mod 3 reduction of the $i$ th Chern class $c_{i} \in H^{2 i}(B U(2) ; \mathbb{Z})$. Since $\wp^{1} c_{2}=c_{1}^{2} c_{2}+c_{2}^{2}$ in $H\left(B U(2) ; \mathbb{F}_{3}\right)$, we see that, as an algebra over the Steenrod algebra $\mathcal{A}(3)$,

$$
H^{*}\left(B L U(2) ; \mathbb{F}_{3}\right) \cong \mathbb{F}_{3}\left[c_{1}, c_{2}\right] \otimes \Lambda\left(\bar{c}_{1}, \bar{c}_{2}\right),
$$

where $\operatorname{deg} \bar{c}_{i}=2 i-1$ and $\wp^{1} \bar{c}_{2}=2 c_{1} c_{2} \bar{c}_{1}+c_{1}^{2} \bar{c}_{2}+2 c_{2} \bar{c}_{2}$.

We now prepare a lemma which is needed in proving Theorems 1.5 and 1.6.

Lemma 3.7. Let $p$ be an odd prime. Under the notation used in the proof of Theorem 1.2 , for any $\widetilde{y}_{i} \in H^{*}\left(G ; \mathbb{F}_{p}\right), \wp^{s} \widetilde{y}_{i}=0$ if $\wp^{s} y_{i}$ is decomposable in $H^{*}\left(B G ; \mathbb{F}_{p}\right)$.

Proof. Let $\left\{\widehat{E}_{r}, \widehat{d}_{r}\right\}$ be the Eilenberg-Moore spectral sequence, which is mentioned in the proof of Theorem 1.2, converging to $H^{*}\left(G ; \mathbb{F}_{p}\right)$. We regard the $E_{2}$-term $\widehat{E}_{2}$ as the torsion product $\operatorname{Tor}_{A \otimes A}\left(\mathbb{F}_{p}, A\right)$ which is obtained from the bar complex $\left(\mathbb{F}_{p} \otimes_{A \otimes A} B^{\bullet}(A \otimes A, A), d\right)$, where $A$ denotes $H^{*}\left(B G ; \mathbb{F}_{p}\right)$. As in the proof of Theorem 1.2 , we have $\wp^{s} \widetilde{y}_{i}=\wp^{s}\left[y_{i} \otimes 1-1 \otimes y_{i}\right]=$ $\left[\wp^{s} y_{i} \otimes 1-1 \otimes \wp^{s} y_{i}\right]$ in Total $\widehat{E}_{0}^{*, *}=H^{*}\left(G ; \mathbb{F}_{p}\right)$. If $z$ is decomposable in $A$, then $[z \otimes 1-1 \otimes z]=0$ in $\widehat{E}_{2}^{*, *}$. In order to verify this, it suffices to show that $[x y \otimes 1-1 \otimes x y]=0$ in $\widehat{E}_{2}^{*, *}$ for any $x, y$ in $A$. We choose an element

$$
u=[y \otimes 1 \mid x \otimes 1-1 \otimes x]-(-1)^{(i+1)(j+1)}[1 \otimes x \mid y \otimes 1-1 \otimes y]
$$

from $\mathbb{F}_{p} \otimes_{A \otimes A} B^{2}(A \otimes A, A)=\mathbb{F}_{p} \otimes \overline{A^{\otimes 2}} \otimes \overline{A^{\otimes 2}} \otimes A$, where $\operatorname{deg} x=i$ and $\operatorname{deg} y=j$. Then we see that

$$
\begin{aligned}
d(u)= & (-1)^{j+1}[y x \otimes 1-y \otimes x] \\
& -(-1)^{(i+1)(j+1)}\left\{(-1)^{i+1}\left[(-1)^{i j} y \otimes x-1 \otimes x y\right]\right\} \\
= & (-1)^{j+1+i j}[x y \otimes 1]+(-1)^{j(i+1)}[1 \otimes x y] \\
= & -(-1)^{j(i+1)}[x y \otimes 1-1 \otimes x y] .
\end{aligned}
$$

Thus the result follows from the fact that $\widehat{E}_{2}^{*, *} \cong \widehat{E}_{\infty}^{*, *} \cong \widehat{E}_{0}^{*, *}$ as $\mathcal{A}(p)$ modules and $\widehat{E}_{0}^{0, *}=0$. 
REMARK 3.8. Suppose that $H^{*}\left(B G ; \mathbb{F}_{2}\right)$ is a polynomial algebra. Then the above argument tells us that the $\mathcal{A}(2)$-module structure on $\widehat{E}_{\infty}^{*, *}=\widehat{E}_{0}^{*, *}$ is determined exactly by the formula $\mathrm{Sq}^{i} \widetilde{y}_{j}=\left[\mathrm{Sq}^{i} y_{j} \otimes 1-1 \otimes \mathrm{Sq}^{i} y_{j}\right]$. More precisely, in $H^{*}\left(G ; \mathbb{F}_{2}\right)$, we see that $\mathrm{Sq}^{i} \widetilde{y}_{j}=\widetilde{y}_{l}$ if $\mathrm{Sq}^{i} y_{j}=y_{l}+\operatorname{decom}$ and $\mathrm{Sq}^{i} \widetilde{y}_{j}=0$ if $\mathrm{Sq}^{i} y_{j}$ is decomposable.

\section{Proofs of Theorems 1.3, 1.5, 1.6 and Proposition 1.4}

Proof of Theorem 1.3. It is immediate to show that (iii) implies (ii). Suppose that condition (ii) holds. It follows from the definition of the module derivation $\mathfrak{D}_{f}^{\prime}$ that the map $q \mathfrak{D}_{f}^{\prime}$ coincides with the composition map

$$
\begin{aligned}
\left(f^{*} \otimes 1\right) \mathfrak{D}: \mathbb{F}_{2}\left[y_{1}, \ldots, y_{l}\right] & \stackrel{\mathfrak{D}}{\longrightarrow} \mathbb{F}_{2}\left[y_{1}, \ldots, y_{l}\right] \otimes \Lambda\left(\bar{y}_{1}, \ldots, \bar{y}_{l}\right) \\
& \stackrel{f^{*} \otimes 1}{\longrightarrow} H^{*}\left(M ; \mathbb{F}_{2}\right) \otimes \Lambda\left(\bar{y}_{1}, \ldots, \bar{y}_{l}\right) \\
& =H H_{*}\left(H^{*}\left(B G ; \mathbb{F}_{2}\right), H^{*}\left(M ; \mathbb{F}_{2}\right)\right) \cong E_{2}^{*, *} \cong E_{\infty}^{*, *} .
\end{aligned}
$$

Observe that the restriction of the map $\left(f^{*} \otimes 1\right) \mathfrak{D}$ to the set $\left\{\mathrm{Sq}^{\operatorname{deg} y_{i}-1} y_{i}\right\}_{y_{i} \in S} \cap\left(\overline{H^{*}} \cdot \overline{H^{*}}\right)$ is the composition map in the condition $C(\mathfrak{D}, f)$.

For any $y_{j} \in S \backslash\left(\operatorname{Im} \mathrm{Sq}^{*} \cap S\right)$, we define an integer $h_{j}$ to be the height of the element $y_{j}$; that is, $\left(\mathrm{Sq}^{*}\right)^{h_{j}} y_{j}=0$ and $\left(\mathrm{Sq}^{*}\right)^{h_{j}-1} y_{j} \neq 0$ modulo decomposable elements. From Remark 3.8 and Definition 2.3(2), we see that

$$
H^{*}\left(G ; \mathbb{F}_{2}\right) \cong \bigotimes_{y_{i} \in S \backslash\left(\operatorname{Im~Sq}^{*} \cap S\right)} \mathbb{F}_{2}\left[\widetilde{y}_{i}\right] /\left(\widetilde{y}_{i}^{h^{h_{i}}}\right)=: A_{2}
$$

as an algebra. Since the map $\left(\operatorname{Im} \mathfrak{D}_{f}^{\prime}\right) \subset F^{-1} H^{*} \stackrel{q}{\rightarrow} E_{0}^{-1, *}$ is a monomorphism, it follows from the condition $C(\mathfrak{D}, f)$ that $\bar{y}_{i}^{2}+\mathfrak{D}_{f}^{\prime} \mathrm{Sq}^{\operatorname{deg} y_{i}-1} y_{i}=\bar{y}_{i}^{2}$ if $\mathrm{Sq}^{\operatorname{deg} y_{i}-1} y_{i}$ is decomposable. Thus we have an isomorphism of $H^{*}\left(M ; \mathbb{F}_{2}\right)$ algebras $\phi: H^{*}\left(P \times_{\text {ad }} G ; \mathbb{F}_{2}\right) \rightarrow A_{2} \otimes H^{*}\left(M ; \mathbb{F}_{2}\right)$ defined by $\phi\left(\bar{y}_{i} \otimes 1\right)=\widetilde{y}_{i} \otimes 1$. Moreover, by considering the morphism of spectral sequences induced by maps in the left face of the diagram (3.6), we get a commutative diagram

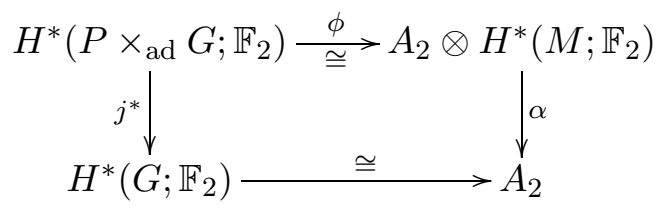

where $\alpha\left(\widetilde{y}_{i} \otimes 1\right)=\widetilde{y}_{i}$ and $\alpha\left(\widetilde{y}_{i} \otimes m\right)=0$ if $\operatorname{deg} m>0$. This allows us to conclude that (ii) implies (i).

We now prove that (i) implies (iii). Suppose that there exists a normalized system $S$ of generators such that the condition $C(\mathfrak{D}, f)$ does not hold. Then there is an element $y_{i}$ in $S$ with the lowest degree such that $\mathrm{Sq}^{\operatorname{deg} y_{i}-1} y_{i} \in \bar{H} \cdot \bar{H}$ and $\mathfrak{D}_{f}^{\prime} \mathrm{Sq}^{\operatorname{deg} y_{i}-1} y_{i} \neq 0$. We can write $\mathfrak{D}_{f}^{\prime} \mathrm{Sq}^{\operatorname{deg} y_{i}-1} y_{i}=$ 
$\sum_{j} \alpha_{j} \bar{y}_{j} \neq 0$, where $\alpha_{j} \in H^{*}\left(M ; \mathbb{F}_{2}\right)$. It follows from the definition of the cohomological splitting that there exists an isomorphism

$$
\phi: H^{*}\left(P \times_{\mathrm{ad}} G ; \mathbb{F}_{2}\right) \rightarrow H^{*}\left(G ; \mathbb{F}_{2}\right) \otimes H^{*}\left(M ; \mathbb{F}_{2}\right) \cong A_{2} \otimes H^{*}\left(M ; \mathbb{F}_{2}\right)
$$

such that $\phi\left(\bar{y}_{i}\right)=\widetilde{y}_{i}+\beta_{i}$, where $\beta_{i}$ is an appropriate element of the ideal in $H^{*}\left(G ; \mathbb{F}_{2}\right) \otimes H^{*}\left(M ; \mathbb{F}_{2}\right)$ generated by $H^{*}\left(M ; \mathbb{F}_{2}\right)^{\geq 1}$, say $\beta_{i}=\sum u_{i k} \widetilde{y}_{1}^{s_{1 i k}} \ldots \widetilde{y}_{l}^{s_{l i k}}$, where $u_{i k} \in H^{*}\left(M ; \mathbb{F}_{2}\right)^{\geq 1}$. From the algebra structure of $H^{*}\left(P \times_{\text {ad }} G ; \mathbb{F}_{2}\right)$ (see Remark 3.4), we have the equality $\bar{y}_{i}^{2}+\sum_{j} \alpha_{j} \bar{y}_{j}=0$. Therefore, in $A_{2} \otimes H^{*}\left(M ; \mathbb{F}_{2}\right)$,

$$
\begin{aligned}
0 & =\phi\left(\bar{y}_{i}^{2}+\sum_{j} \alpha_{j} \bar{y}_{j}\right)=\widetilde{y}_{i}^{2}+\beta_{i}^{2}+\sum_{j} \alpha_{j}\left(\widetilde{y}_{j}+\beta_{j}\right) \\
& =\sum_{j} \alpha_{j} \widetilde{y}_{j}+\left(\sum u_{i k} \widetilde{y}_{1}^{s_{1 i k}} \ldots \widetilde{y}_{l}^{s_{l i k}}\right)^{2}+\sum_{j} \alpha_{j}\left(\sum u_{j k} \widetilde{y}_{1}^{s_{1 j k}} \ldots \widetilde{y}_{l}^{s_{l j k}}\right) .
\end{aligned}
$$

Observe that $\widetilde{y}_{i}^{2}=0$ in $A_{2}$ as $\mathrm{Sq}^{\operatorname{deg} y_{i}-1} y_{i}$ is decomposable (Remark 3.8). Let $\alpha_{s}$ be of the lowest degree among the non-zero elements $\alpha_{1}, \ldots, \alpha_{l}$. Looking at the element $\alpha_{s} \widetilde{y}_{s}$, we see that the above equality contradicts the set $\left\{\widetilde{y}_{1}^{s_{1}} \ldots \widetilde{y}_{l}^{s_{l}}: s_{i} \leq 2^{h_{i}}\right\}$ being an $H^{*}\left(M ; \mathbb{F}_{2}\right)$-basis of $A_{2} \otimes H^{*}\left(M ; \mathbb{F}_{2}\right)$.

In order to prove Proposition 1.4, we prepare a lemma, which also plays an important role in the proof of Theorem 1.6.

Lemma 4.1. Let $G(\alpha, m)$ denote the special orthogonal group $S O(m)$, the special unitary group $S U(m)$ or the symplectic group $S p(m)$ according as $\alpha=1,2$ or 4 . Let $y_{i}^{(1)}, y_{i}^{(2)}$ and $y_{i}^{(4)}$ be the ith Stiefel-Whitney class, Chern class and symplectic Pontryagin class, respectively. Then

$$
\mathrm{Sq}^{\alpha(i-1)}\left(y_{i}^{(\alpha)}+\operatorname{decom}\right) \equiv \mathrm{Sq}^{\alpha(i-1)} y_{i}^{(\alpha)}
$$

modulo the ideal $\left(\overline{H^{*}} \cdot \overline{H^{*}} \cdot \overline{H^{*}}\right)$ of $H^{*}\left(B G(\alpha, m) ; \mathbb{F}_{2}\right)$, where

$$
\overline{H^{*}}=\bigoplus_{i \geq 1} H^{i}\left(B G(\alpha, m) ; \mathbb{F}_{2}\right) .
$$

Proof. We prove that

$$
\mathrm{Sq}^{i-1}\left(w_{i}+\sum_{1 \leq k \leq(i-1) / 2} a_{k} w_{i-k} w_{k}+W\right) \equiv \mathrm{Sq}^{i-1} w_{i}
$$

modulo the ideal $I=\left(\overline{H^{*}} \cdot \overline{H^{*}} \cdot \overline{H^{*}}\right)$, where $W$ is some element in $I$ and $w_{i}$ denotes the $i$ th Stiefel-Whitney class. To this end, it suffices to show that $\mathrm{Sq}^{i-1}\left(w_{i-k} w_{k}\right) \in I$. This follows from the Cartan formula:

$$
\begin{aligned}
\mathrm{Sq}^{i-1}\left(w_{i-k} w_{k}\right) & =\sum_{l+m=i-1} \mathrm{Sq}^{l} w_{i-k} \mathrm{Sq}^{m} w_{k} \\
& =\mathrm{Sq}^{i-k} w_{i-k} \mathrm{Sq}^{k-1} w_{k}+\mathrm{Sq}^{i-k-1} w_{i-k} \mathrm{Sq}^{k} w_{k} \in I .
\end{aligned}
$$


The same argument works for $\alpha=2$ and 4 . The details are left to the reader.

Proof of Proposition 1.4. We choose a classifying map $f$ of a given $S O(n)$-bundle. Theorem 1.3 implies that the adjoint bundle splits on mod 2 cohomology if $f^{*}$ is trivial.

We prove the "only if" part. It follows from the Wu formula that $\mathrm{Sq}^{i-1} w_{i}$ $=w_{2 i-1}+$ decom, where $w_{i}$ is the $i$ th Stiefel-Whitney class, $2 i-1 \leq n$ and decom denotes an appropriate decomposable element in $H^{*}\left(B S O(n) ; \mathbb{F}_{2}\right)$. This implies that the set $\left\{y_{(i, s)}\right\}_{(i, s) \in B}$ defined by

$$
\begin{gathered}
y_{(i,-1)}=w_{i}, y_{(i, s)}=\mathrm{Sq}^{2^{s}(i-1)} \ldots \mathrm{Sq}^{2(i-1)} \mathrm{Sq}^{(i-1)} w_{i} \quad(s \geq 0), \\
B=\left\{(i, s) \mid i \text { is even, } s \geq-1,2^{s+1}(i-1)+1 \leq n\right\}
\end{gathered}
$$

is a normalized system of generators of $H^{*}\left(B S O(n) ; \mathbb{F}_{2}\right)$. Observe that $y_{(i, s)}=w_{2^{s+1}(i-1)+1}+$ decom. We shall write $\varepsilon\left(2^{s+1}(i-1)+1\right)$ for the pair $(i, s)$ corresponding to the integer $2^{s+1}(i-1)+1$.

Assume that $f^{*}$ is not trivial. If $n$ is odd, let $j$ be the integer such that $2 j-1=n+2$. From the $\mathrm{Wu}$ formula and Lemma 4.1, we conclude that

$$
\begin{aligned}
\mathrm{Sq}^{j-1} y_{\varepsilon(j)} & =\mathrm{Sq}^{j-1} w_{j}+\beta \\
& =w_{n} w_{2}+w_{n-1} w_{3}+\ldots+w_{j} w_{j-1}+\beta \\
& =y_{\varepsilon(n)} y_{\varepsilon(2)}+y_{\varepsilon(n-1)} y_{\varepsilon(3)}+\ldots+y_{\varepsilon(j)} y_{\varepsilon(j-1)}+\beta^{\prime},
\end{aligned}
$$

where $\beta$ and $\beta^{\prime}$ are in the ideal $\left(\overline{H^{*}} \cdot \overline{H^{*}} \cdot \overline{H^{*}}\right)$. Since there exists $y_{\varepsilon(l)}$ such that $f^{*}\left(y_{\varepsilon(l)}\right) \neq 0$ and $f^{*}\left(y_{\varepsilon(j)}\right)=0$ for any $j<l$, it follows that $f^{*} \otimes 1\left(y_{\varepsilon(l)} \otimes \bar{y}_{\varepsilon(2 j-1-l)}\right) \neq 0$ in $\Lambda\left(\bar{y}_{(i, s)} ;(i, s) \in B\right)^{2 j-1-l-1} \otimes H^{l}\left(M ; \mathbb{F}_{2}\right)$ and hence $f^{*} \otimes 1 \circ \mathfrak{D}\left(\mathrm{Sq}^{j-1} y_{\varepsilon(j)}\right) \neq 0$ in $H H_{*}\left(H^{*}\left(B S O(n) ; \mathbb{F}_{2}\right), H^{*}\left(M ; \mathbb{F}_{2}\right)\right)$. Theorem 1.3 implies that the associated bundle does not split on mod 2 cohomology.

If $n$ is even, let $j$ and $k$ be the integers such that $2 j-1=n+1$ and $2 k-1=n+3$. Using the $\mathrm{Wu}$ formula, we obtain the equalities

$$
\begin{aligned}
& \mathrm{Sq}^{j-1} w_{j}=w_{n-1} w_{2}+w_{n-2} w_{3}+\ldots+w_{i} w_{i-1}, \\
& \mathrm{Sq}^{k-1} w_{k}=w_{n} w_{3}+w_{n-1} w_{4}+\ldots+w_{k} w_{k-1} .
\end{aligned}
$$

The same argument as above shows that $f^{*} \otimes 1 \circ \mathfrak{D}\left(\mathrm{Sq}^{l-1} y_{\varepsilon(l)}\right) \neq 0(l=j$ or $k)$ in $H H_{*}\left(H^{*}\left(B S O(n) ; \mathbb{F}_{2}\right), H^{*}\left(M ; \mathbb{F}_{2}\right)\right)$. This completes the proof.

Combining Proposition 1.4 with Theorem 3.1 and Remark 3.4, we can easily establish the following proposition.

Proposition 4.2. The adjoint bundle $P \times_{\text {ad }} S O(n) \rightarrow M$ splits on mod 2 cohomology if and only if the bundle admits an $\mathcal{A}(2)$-cohomological splitting. 
We are now ready to prove Theorem 1.5.

Proof of Theorem 1.5. It is not hard to prove the "if" part by applying Theorem 3.1 because the Steenrod operations act trivially on the generators of $H^{*}\left(B G ; \mathbb{F}_{p}\right)$ except for $\wp^{1}$.

Assume that the bundle $G \stackrel{j}{\rightarrow} B L G \rightarrow B G$ admits an $\mathcal{A}(p)$-cohomological splitting and that $H^{*}\left(G ; \mathbb{F}_{p}\right)$ is not isomorphic to the $\bmod p$ cohomology of any torus. From $\left[2\right.$, Theorem 1] and [9, Theorem 1.1], we see that $H^{*}\left(G ; \mathbb{F}_{p}\right)$ is not cocommutative if $H^{*}(G ; \mathbb{Z})$ has $p$-torsion. Consider the cobar type Eilenberg-Moore spectral sequence $\left\{E_{r}, d_{r}\right\}$ converging to $H^{*}\left(B L G ; \mathbb{F}_{p}\right)$ with

$$
E_{2}^{*, *} \cong \operatorname{Cotor}_{H^{*}\left(G ; \mathbb{F}_{p}\right)}\left(\mathbb{F}_{p}, H^{*}\left(G ; \mathbb{F}_{p}\right)\right),
$$

where the $H^{*}\left(G ; \mathbb{F}_{p}\right)$-comodule structure of $H^{*}\left(G ; \mathbb{F}_{p}\right)$ is induced by the adjoint action on $G$. Then the fact that $H^{*}\left(G ; \mathbb{F}_{p}\right)$ is not cocommutative implies that the edge homomorphism of the spectral sequence, which coincides with $j^{*}$, is not surjective (for details of this argument, see [12, Remark 1.6]). It follows that $H^{*}(G ; \mathbb{Z})$ actually has no $p$-torsion. Thus the rational cohomological data enables us to deduce that $H^{*}\left(G ; \mathbb{F}_{p}\right) \cong \Lambda\left(x_{2 m_{1}-1}, \ldots, x_{2 m_{l}-1}\right)$ and $H^{*}\left(B G ; \mathbb{F}_{p}\right) \cong \mathbb{F}_{p}\left[y_{2 m_{1}}, \ldots, y_{2 m_{l}}\right]$ as algebras.

By assumption, there is the least integer $m_{s}$ of $m_{1}, \ldots, m_{l}$ such that $\left(m_{s}, p\right)=1$; that is, $1=m_{1}=\ldots=m_{s-1}<m_{s} \leq \ldots \leq m_{l}$. From the Adem relation $(m+1) \wp^{m+1}=\wp^{1} \wp^{m}$, we see that $\wp^{1} \wp^{m_{s}-1} y_{2 m_{s}}=m_{s} \wp^{m_{s}} y_{2 m_{s}}=$ $m_{s} y_{2 m_{s}}^{p} \neq 0$ and hence $\wp^{m_{s}-1} y_{2 m_{s}} \neq 0$. Suppose that $\wp^{m_{s}-1} y_{2 m_{s}}$ is indecomposable. Let $y_{2 m_{j}}$ be an indecomposable factor of the summation representing $\wp^{m_{s}-1} y_{2 m_{s}}$. Then the integer $m_{j}=p\left(m_{s}-1\right)+1$ is prime to $p$. Thus we can find an integer $m_{k}\left(\geq m_{s}\right)$ prime to $p$ such that $\wp^{m_{k}-1} y_{2 m_{k}}$ is a nonzero decomposable element. Without loss of generality, we can assume that $\wp^{m_{s}-1} y_{2 m_{s}}=y_{2 m_{j_{1}}}, \wp^{m_{j_{1}}-1} y_{2 m_{j_{1}}}=y_{2 m_{j_{2}}}, \ldots$, and $\wp^{m_{j_{t}}-1} y_{2 m_{j_{t}}}=y_{2 m_{k}}$. Observe that

$$
y_{2 m_{k}}=\wp^{p^{l-1}\left(m_{s}-1\right)} \ldots \wp \wp^{p\left(m_{s}-1\right)} \wp^{m_{s}-1} y_{2 m_{s}}
$$

for some $l$ and $m_{k}=\left(m_{s}-1\right) p^{l}+1$. Since $\wp^{1} \wp^{m_{k}-1} y_{2 m_{k}}=m_{k} \wp^{m_{k}} y_{2 m_{k}}=$ $m_{k} y_{2 m_{k}}^{p}$, it follows that, in a term of $\wp^{m_{k}-1} y_{2 m_{k}}$, there exists a factor $y_{2 m_{a}}$ whose power is prime to $p$ such that $\wp^{1} y_{2 m_{a}}=\alpha y_{2 m_{k}}^{j}$ for some $j$ and non-zero integer $\alpha$. Thus we can write

$$
\wp^{m_{k}-1} y_{2 m_{k}}=P_{1} y_{2 m_{a}}^{u_{1}}+P_{2} y_{2 m_{a}}^{u_{2}}+\ldots+P_{q} y_{2 m_{a}}^{u_{q}}+P_{q+1},
$$

where $P_{j}$ denotes an appropriate non-zero polynomial which does not contain $y_{2 m_{a}}$ as a factor and $\left(u_{j}, p\right)=1$ for some $j$ and $u_{1}>\ldots>u_{q}$. We assume that $u_{b}$ is the largest integer of $u_{1}, \ldots, u_{q}$ which is prime to $p$. Let $\phi: H^{*}\left(B L G ; \mathbb{F}_{p}\right) \stackrel{\cong}{\longrightarrow} H^{*}\left(B G ; \mathbb{F}_{p}\right) \otimes H^{*}\left(G ; \mathbb{F}_{p}\right)$ be the isomorphism which gives an $\mathcal{A}(p)$-cohomological splitting. Under the same notation as in the proof of Theorem 1.2, we have a commutative diagram of $\mathcal{A}(p)$-algebras: 


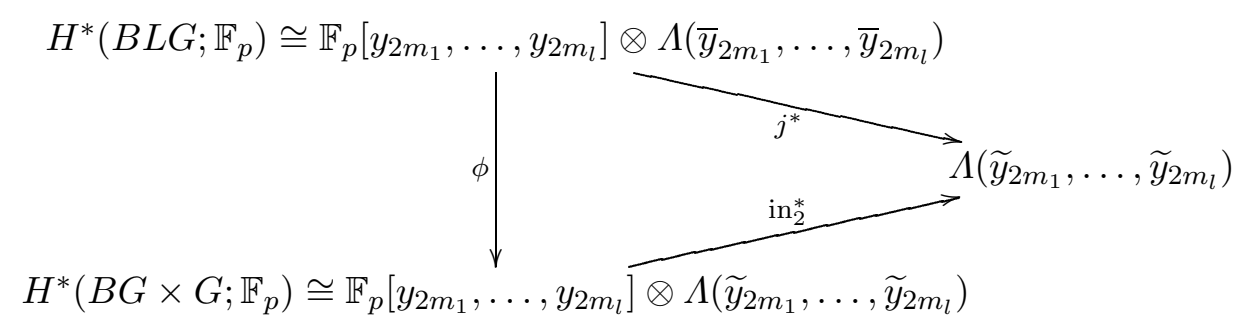

For dimensional reasons, we have $\phi\left(\bar{y}_{2 m_{i}}\right)=\widetilde{y}_{2 m_{i}}$ for $1 \leq i \leq s-1$. Since $\operatorname{deg} \phi\left(\bar{y}_{2 m_{s}}\right)$ is odd, it follows that $\phi\left(\bar{y}_{2 m_{s}}\right)=\widetilde{y}_{2 m_{s}}+P$, where $P$ is a polynomial containing at least one element $\widetilde{y}_{i}$ as a factor in each term. Thus we see that

$$
\phi\left(\bar{y}_{2 m_{1}} \ldots \bar{y}_{2 m_{s-1}} \bar{y}_{2 m_{s}}\right)=\widetilde{y}_{2 m_{1}} \ldots \widetilde{y}_{2 m_{s-1}} \widetilde{y}_{2 m_{s}} .
$$

Apply the Steenrod operation $\wp^{m_{k}-1} \ldots \wp^{m_{s}-1}$ to the right hand side. Then it follows from Lemma 3.7 that

$$
\wp^{m_{k}-1} \ldots \wp^{m_{s}-1} \widetilde{y}_{2 m_{1}} \ldots \widetilde{y}_{2 m_{s-1}} \widetilde{y}_{2 m_{s}}=0 .
$$

Observe that $\wp^{n} \widetilde{y}_{2 m_{i}}=0$ for $1 \leq i \leq s-1$ if $n \neq 0$. On the other hand, from Theorem 3.1, we have

$$
\begin{aligned}
\wp^{p^{l-1}\left(m_{s}-1\right)} \ldots \wp^{p\left(m_{s}-1\right)} \wp^{m_{s}-1} \bar{y}_{2 m_{1}} \ldots \bar{y}_{2 m_{s-1}} \bar{y}_{2 m_{s}} \\
=\wp^{m_{k}-1} \ldots \wp^{m_{s}-1} \bar{y}_{2 m_{1}} \ldots \bar{y}_{2 m_{s-1}} \bar{y}_{2 m_{s}}=\bar{y}_{2 m_{1}} \ldots \bar{y}_{2 m_{s-1}} \wp^{m_{k}-1} \bar{y}_{2 m_{k}} \\
=\bar{y}_{2 m_{1}} \ldots \bar{y}_{2 m_{s-1}} \mathfrak{D}^{\prime}\left(\sum_{j=1}^{q+1} P_{j} y_{2 m_{a}}^{u_{j}}\right)=\bar{y}_{2 m_{1}} \ldots \bar{y}_{2 m_{s-1}}\left(\sum_{j=1}^{q+1}\left(\mathfrak{D}^{\prime} P_{j}\right) y_{2 m_{a}}^{u_{j}}\right) \\
\quad+\bar{y}_{2 m_{1}} \ldots \bar{y}_{2 m_{s-1}} P_{b} \cdot u_{b} \cdot y_{2 m_{a}}^{u_{b}-1} \bar{y}_{2 m_{a}}+\ldots \\
\quad+\bar{y}_{2 m_{1}} \ldots \bar{y}_{2 m_{s-1}} P_{q} \cdot u_{q} \cdot y_{2 m_{a}}^{u_{q}-1} \bar{y}_{2 m_{a}} .
\end{aligned}
$$

Looking at the term $\bar{y}_{2 m_{1}} \ldots \bar{y}_{2 m_{s-1}} P_{b} \cdot u_{b} \cdot y_{2 m_{a}}^{u_{b}-1} \bar{y}_{2 m_{a}}$, we see that

$$
\wp^{m_{k}-1} \ldots \wp^{m_{s}-1} \bar{y}_{2 m_{1}} \ldots \bar{y}_{2 m_{s-1}} \bar{y}_{2 m_{s}} \neq 0
$$

in $H^{*}\left(B L G ; \mathbb{F}_{p}\right)$, which is a contradiction. This completes the proof.

Proof of Theorem 1.6. If $p=2$ and $G$ is a fake Lie group of type $S^{3}$, then an explicit calculation applying Theorem 3.1 enables us to deduce that the bundle $B L G \rightarrow B G$ admits an $\mathcal{A}(2)$-cohomological splitting.

We proceed to the proof of the "only if" part. Since $H$ is simply connected, Theorem 1.5 implies that $p=2$. Assume that $B L G \rightarrow B G$ admits an $\mathcal{A}(2)$-cohomological splitting and $G$ is a fake Lie group of type $H$ which is not $S^{3}$. From [8] or [12, Proposition 4.1], it follows that $H^{*}(G ; \mathbb{Z})$ has no 2 -torsion and hence $G$ is of type $S U(m)(m>2)$ or $S p(n)(n>1)$. We choose an integer $k$ so that $2^{k}+2 \leq m<2^{k+1}+2$. Lemma 4.1 and the $\mathrm{Wu}$ 
formula yield

$$
\begin{aligned}
\mathrm{Sq}^{2^{k-1}} \ldots \mathrm{Sq}^{2} c_{2} & =c_{2^{k-1}+1}+\text { decom } \\
\mathrm{Sq}^{2^{k}} \mathrm{Sq}^{2^{k-1}} \ldots \mathrm{Sq}^{2} c_{2} & =\mathrm{Sq}^{2^{k}} c_{2^{k-1}+1}+\beta \\
& =\sum_{i=2}^{2^{k-1}} c_{2^{k}+1-i} c_{i}+\beta,
\end{aligned}
$$

in $H^{*}\left(B S U(m) ; \mathbb{F}_{2}\right)$, where $\beta \in\left(\overline{H^{*}} \cdot \overline{H^{*}} \cdot \overline{H^{*}}\right)$. Since

$$
\mathrm{Sq}^{2^{k}} \ldots \mathrm{Sq}^{2} \bar{y}_{2}=\mathfrak{D}^{\prime}\left(\mathrm{Sq}^{2^{k}} \ldots \mathrm{Sq}^{2} y_{2}\right)=\mathfrak{D}^{\prime}\left(\sum_{i=2}^{2^{k-1}} c_{2^{k}+1-i} c_{i}+\beta\right) \neq 0
$$

in $H^{*}\left(B L S U(m) ; \mathbb{F}_{2}\right)$, as in the proof of Theorem 1.5, it follows that $B L S U(m) \rightarrow B S U(m)$ does not admit an $\mathcal{A}(2)$-cohomological splitting, which is a contradiction. The same argument works in the case $G=S p(m)$, where we use an integer $s$ with $2^{s}+s+2 \leq m<4\left(2^{s+1}+s+3\right)$ and the element $\mathrm{Sq}^{4\left(2^{s-1}+1\right)} \ldots \mathrm{Sq}^{4 \cdot 2} \mathrm{Sq}^{4} q_{2}$ instead of the integer $k$ and the element $\mathrm{Sq}^{2^{k-1}} \ldots \mathrm{Sq}^{2} c_{2}$.

Proof of Corollary 1.7. Choose a prime number $p$ so that $H^{*}(G ; \mathbb{Z})$ has no $p$-torsion. By the same argument as in the proof of Theorem 1.5, we see that $H^{*}\left(B L G ; \mathbb{F}_{p}\right)$ is not isomorphic to $H^{*}\left(B G \times G ; \mathbb{F}_{p}\right)$ as an $\mathcal{A}(p)$-module. Observe that it is not necessary to apply the factor $\bar{y}_{2 m_{1}} \ldots \bar{y}_{2 m_{s-1}}$ in the proof because $H^{1}\left(G ; \mathbb{F}_{p}\right)=0$. Thus we have the result.

5. Homotopy types of $S U(n)$-adjoint bundles. Let $F \rightarrow E \rightarrow M$ and $F \rightarrow E^{\prime} \rightarrow M$ be fibrations with the same base and fibre. They are said to be rationally homotopically equivalent if there exists a homotopy equivalence $E_{\mathbb{Q}} \stackrel{\simeq}{\rightarrow} E_{\mathbb{Q}}^{\prime}$ which covers the identity map of $M_{\mathbb{Q}}$. If we classify $G$-adjoint bundles over a connected space under rational homotopy equivalence of fibrations, then the set of equivalence classes consists of just one element which has the trivial bundle as a representative element. This follows from Theorem 1.2 and [6, Theorem II]. In contrast with the rational case, Theorem 1.8 asserts that, in some cases, the homotopical classification of bundles is equivalent to the rigid topological classification.

Proof of Theorem 1.8. Let $M$ be a connected 4- or 5-dimensional CW complex such that $H^{4}(M ; \mathbb{Z}) \cong \mathbb{Z} / p$. Since $\pi_{5}(B S U(n))=\pi_{4}(S U(n))=0$, it follows that there is a bijection $[M, B S U] \rightarrow[M, K(\mathbb{Z}, 4)]$. Thus the principal $S U(n)$-bundles over $M$ are classified by elements $f^{*}\left(c_{2}\right)$ in $H^{4}(M ; \mathbb{Z})$ which are characterized by the homotopy classes of classifying maps and the second Chern class. 
Let $P_{m} \rightarrow M$ be an $S U(n)$-bundle representing the class $f^{*}\left(c_{2}\right)=m \iota$, where $\iota$ is a generator of $H^{4}(M ; \mathbb{Z})$. For any odd prime $p$, the $\bmod p \mathrm{Wu}$ formula for Steenrod operations on $H^{*}\left(B U ; \mathbb{F}_{p}\right)$ (see [14]) tells us that

$$
\wp^{1}\left(c_{2}\right)=-\frac{1}{2} c_{2} c_{p-1}+\varrho
$$

on $H^{*}\left(B S U(n) ; \mathbb{F}_{p}\right)$, where $\varrho$ denotes a linear combination of elements which are different from $\alpha c_{2} c_{p-1}\left(\alpha \in \mathbb{F}_{p}\right)$. Observe that $\varrho$ is decomposable; that is, $\varrho$ does not have the element $\gamma c_{p+1}\left(\gamma \in \mathbb{F}_{p}\right)$ as a term because $n=p-1$ or $p$. Suppose that the $S U(n)$-adjoint bundles $S U(n) \stackrel{j}{\rightarrow} P_{l} \times_{\text {ad }} S U(n) \rightarrow M$ and $S U(n) \stackrel{j^{\prime}}{\rightarrow} P_{m} \times$ ad $S U(n) \rightarrow M$ are homotopy equivalent in the sense mentioned in Section 1. Then there exists a map $\phi: P_{m} \times_{\text {ad }} S U(n) \rightarrow$ $P_{l} \times_{\text {ad }} S U(n)$ such that the induced map $\phi^{*}: H^{*}\left(P_{l} \times_{\text {ad }} S U(n) ; \mathbb{F}_{p}\right) \rightarrow$ $H^{*}\left(P_{m} \times\right.$ ad $\left.S U(n) ; \mathbb{F}_{p}\right)$ is an isomorphism of $H^{*}\left(M ; \mathbb{F}_{p}\right)$-algebras with $j^{\prime *} \phi^{*}$ $=j^{*}$. Since $H^{*}\left(B S U(n) ; \mathbb{F}_{p}\right)$ is a polynomial algebra generated by finitely many Chern classes, the same argument as in the proof of Theorem 1.2 works to prove that

$$
\begin{aligned}
H^{*}\left(P_{l} \times_{\text {ad }} S U(n) ; \mathbb{F}_{p}\right) & \cong H^{*}\left(P_{m} \times_{\text {ad }} S U(n) ; \mathbb{F}_{p}\right) \\
& \cong H^{*}\left(M ; \mathbb{F}_{p}\right) \otimes \Lambda\left(\bar{c}_{2}, \bar{c}_{3}, \ldots, \bar{c}_{n}\right)
\end{aligned}
$$

as $H^{*}\left(M ; \mathbb{F}_{p}\right)$-algebras. Thus we can write $\phi^{*}\left(\bar{c}_{2}\right)=\bar{c}_{2}+\eta$ for some $\eta \in$ $H^{3}\left(M ; \mathbb{F}_{p}\right)$. Since $f^{*}\left(c_{j}\right)=0$ if $j \neq 2$ for the classifying maps $f$ of the principal bundles $P_{l} \rightarrow M$ and $P_{m} \rightarrow M$, we see that $\mathfrak{D}_{f}^{\prime} \varrho=0$. Theorem 3.1 enables us to deduce that $\wp^{1}\left(\bar{c}_{2}\right)=-\frac{1}{2} l \iota \bar{c}_{p-1}$ in $H^{*}\left(P_{l} \times_{\text {ad }} S U(n) ; \mathbb{F}_{p}\right)$ and $\wp^{1}\left(\bar{c}_{2}\right)=-\frac{1}{2} m \iota \bar{c}_{p-1}$ in $H^{*}\left(P_{m} \times_{\text {ad }} S U(n) ; \mathbb{F}_{p}\right)$ if $p \neq 3$. The element $\phi^{*}\left(\bar{c}_{p+1}\right)$ can be expressed as $\bar{c}_{p-1}+Q$, where $Q \in \sum_{i=1}^{4} H^{i}\left(M ; \mathbb{F}_{p}\right) \cdot \Lambda\left(\bar{c}_{2}, \ldots, \bar{c}_{q}, \ldots\right)$. Therefore we have

$$
\begin{aligned}
& \phi^{*} \wp^{1}\left(\bar{c}_{2}\right)=-\frac{1}{2} l \iota\left(\bar{c}_{p-1}+Q\right)=-\frac{1}{2} l \iota \bar{c}_{p-1}, \\
& \wp^{1} \phi^{*}\left(\bar{c}_{2}\right)=\wp^{1}\left(\bar{c}_{2}+\eta\right)=\wp^{1}\left(\bar{c}_{2}\right)=-\frac{1}{2} m \iota \bar{c}_{p-1} .
\end{aligned}
$$

Hence $m \equiv l \bmod p$.

For $p=3$, we have $\wp^{1}\left(\bar{c}_{2}\right)=2 m \iota \bar{c}_{p-1}$ in $H^{*}\left(P_{m} \times_{\text {ad }} S U(n) ; \mathbb{F}_{3}\right)$ and $\wp^{1}\left(\bar{c}_{2}\right)=2 l \iota \bar{c}_{p-1}$ in $H^{*}\left(P_{l} \times{ }_{\text {ad }} S U(n) ; \mathbb{F}_{3}\right)$. Applying the same argument as above, we can deduce that $m \equiv l \bmod 3$. This completes the proof.

6. Conclusion. In our consideration of the cohomology algebra $H^{*}\left(P \times_{\text {ad }} G ; \mathbb{F}_{p}\right)$ over $\mathcal{A}(p)$, the condition that $H^{*}\left(B G ; \mathbb{F}_{p}\right)$ is a polynomial algebra has been needed as input data of the Eilenberg-Moore spectral sequence. However, we expect that knowledge of $H^{*}\left(B G ; \mathbb{F}_{p}\right)$ for degrees less than some integer brings us partial information about $H^{*}\left(P \times_{\text {ad }} G ; \mathbb{F}_{p}\right)$. This information may be useful in classifying the adjoint bundles homotopically even if $H^{*}\left(B G ; \mathbb{F}_{p}\right)$ is not a polynomial algebra. 
The module derivation we have defined in Section 2 still works on the Eilenberg-Moore spectral sequence for the homotopy fibre square with the diagonal map $\Delta: X \rightarrow X \times X$ in the corner. Therefore when the EilenbergMoore spectral sequence is applied to the homotopy fibre square

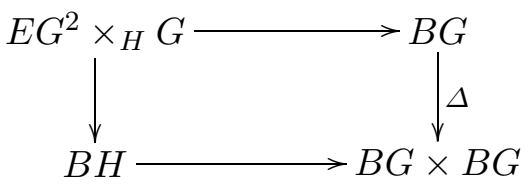

(see Section 3), we also expect our module derivation to be of use in studying the action of the Steenrod operations on the cohomology algebras of homogeneous spaces, biquotient spaces, which have been studied in [5], [15], and of spaces of the form $E G \times{ }_{K} G / H$, where $K$ and $H$ are subgroups of a finite loop space $G$. We hope to present such considerations using module derivations in a forthcoming paper.

Acknowledgements. The second author would like to thank JeanClaude Thomas and Université d'Angers, where a preliminary version of this article has been written, for their warm hospitality. We also thank the referee for helpful comments on improving the proof of Theorem 3.1.

\section{References}

[1] M. Bökstedt and I. Ottosen, Homotopy orbits of free loop spaces, Fund. Math. 162 (1999), 251-275.

[2] W. Browder, Homology ring of groups, Amer. J. Math. 90 (1968), 318-333.

[3] N. Castellana and N. Kitchloo, A homotopy construction of the adjoint representation for Lie groups, Math. Proc. Cambridge Philos. Soc. 133 (2002), 399-409.

[4] J.-H. Eschenburg, New examples of manifolds with strictly positive curvature, Invent. Math. 66 (1982), 469-480.

[5] - Cohomology of biquotients, Manuscripta Math. 75 (1992), 151-166.

[6] S. Halperin and J.-C. Thomas, Rational equivalence of fibrations with fibre $G / K$, Canad. J. Math. 34 (1982), 31-43.

[7] N. Iwase, Adjoint action of finite loop space, Proc. Amer. Math. Soc. 125 (1997), $2753-2757$.

[8] N. Iwase and A. Kono, Adjoint action of a finite loop space II, Proc. Roy. Soc. Edinburgh Sect. A 129 (1999), 773-785.

[9] R. Kane, Primitivity and finite H-spaces, Quart. J. Math. Oxford Ser. (2) 26 (1975), 309-313.

[10] A. Kono and K. Kozima, The adjoint action of a Lie group on the space of loops, J. Math. Soc. Japan 45 (1993), 495-510.

[11] K. Kuribayashi, On the mod p cohomology of spaces of free loops on the Grassmann and Stiefel manifolds, J. Math. Soc. Japan 43 (1991), 331-346.

[12] -, Module derivations and the adjoint action of a finite loop space, J. Math. Kyoto Univ. 39 (1999), 67-85. 
[13] D. Notbohm and L. Smith, Fake Lie groups and maximal tori, I, Math. Ann. 288 (1990), 637-661.

[14] P. B. Shay, Mod $p$ Wu formulas for the Steenrod algebra and the Dyer-Lashof algebra, Proc. Amer. Math. Soc. 63 (1977), 339-347.

[15] W. Singhof, On the topology of double coset manifolds, Math. Ann. 297 (1993), 133-146.

[16] L. Smith, On the Künneth theorem I, Math. Z. 166 (1970), 94-140.

[17] -, On the characteristic zero cohomology of the free loop space, Amer. J. Math. 103 (1981), 887-910.

[18] S. Tsukuda, On isomorphism classes of gauge groups, Topology Appl. 87 (1998), $173-187$.

Department of Mathematics

Faculty of Science

Kyoto University

Kyoto 606, Japan

E-mail: kono@kusm.kyoto-u.ac.jp
Department of Applied Mathematics Faculty of Science

Okayama University of Science Okayama 700-0005, Japan E-mail: kuri@geom.xmath.ous.ac.jp

Received 1 December 2002;

in revised form 5 January 2004 